\title{
When Keeping in Mind Supports Later Bringing to Mind: Neural Markers of Phonological Rehearsal Predict Subsequent Remembering
}

\author{
Lila Davachi ${ }^{1}$, Anat Maril ${ }^{2}$, and Anthony D. Wagner ${ }^{1,3}$
}

\begin{abstract}
The ability to bring to mind a past experience depends on the cognitive and neural processes that are engaged during the experience and that support memory formation. A central and much debated question is whether the processes that underlie rote verbal rehearsal-that is, working memory mechanisms that keep information in mind-impact memory formation and subsequent remembering. The present study used eventrelated functional magnetic resonance imaging (fMRI) to explore the relation between working memory maintenance operations and long-term memory. Specifically, we investigated whether the magnitude of activation in neural regions supporting the on-line maintenance of verbal codes is predictive of subsequent memory for words that were roterehearsed during learning. Furthermore, during rote rehearsal, the extent of neural activation in regions associated with
\end{abstract}

\section{INTRODUCTION}

Why some experiences are remembered whereas others are forgotten has long been a central question in the study of memory. Beginning with the influential work of Ebbinghaus (1885/1964), theorists have generally agreed that the processes engaged during an experience contribute to whether the experience will be memorable. There has been much debate, however, regarding the nature of the processes that are thought to support episodic encoding, that is, the transformation of an experience into a durable memory representation such that the experience can be subsequently consciously remembered (Tulving, 1983). At the center of this debate has been whether short-term or working memory processes that subserve item maintenance-that is, keeping information in mind-contribute to encoding such that the item can be subsequently rememberedthat is, later bringing information to mind (Baddeley, 1998; Bjork, 1975; Craik \& Lockhart, 1972; Atkinson \& Shiffrin, 1968).

\footnotetext{
${ }^{1}$ Massachusets Institute of Technology, ${ }^{2}$ Harvard University,

${ }^{3} \mathrm{MGH}-\mathrm{NMR}$ Center, Charlestown, MA
}

semantic retrieval was assessed to determine the role that incidental semantic elaboration may play in subsequent memory for rote-rehearsed items. Results revealed that (a) the magnitude of activation in neural regions previously associated with phonological rehearsal (left prefrontal, bilateral parietal, supplementary motor, and cerebellar regions) was correlated with subsequent memory, and (b) while rote rehearsal did not-on average-elicit activation in an anterior left prefrontal region associated with semantic retrieval, activation in this region was greater for trials that were subsequently better remembered. Contrary to the prevalent view that rote rehearsal does not impact learning, these data suggest that phonological maintenance mechanisms, in addition to semantic elaboration, support the encoding of an experience such that it can be later remembered.

According to one early, but influential, model of memory (Atkinson \& Shiffrin, 1968), the extent of long-term memory formation directly relates to the degree to which an item is maintained in working memory. From this perspective, duration in working memory was thought to determine the degree of transfer to long-term memory. For example, as an item, such as a telephone number, is rehearsed in working memory, the processes that support maintenance of that item or rote rehearsal also serve to contribute to memory formation. Evidence for this hypothesis initially derived from observations that the more an item is rehearsed, the greater the likelihood it has of being later remembered (Woodward, Bjork, \& Jongeward, 1973; Rundus, 1971). For example, Rundus (1971) demonstrated a correlation between the number of times an item was rehearsed and the probability that it was subsequently recalled at test.

Significant challenges to the hypothesis that maintenance in working memory yields long-term encoding subsequently emerged from two lines of empirical work. First, in contrast to initial findings, subsequent studies failed to demonstrate that rote rehearsal increased the probability of an item's later recall (Craik \& Watkins, 
1973; Woodward et al., 1973). Second, unambiguous evidence was garnered indicating that the probability of later recall markedly depends on the type, or level, of processing performed during an experience (Craik \& Lockhart, 1972). Holding processing time constant, "deeper" semantic processing (also termed "elaborative rehearsal") yields superior subsequent memory relative to "shallower" nonsemantic processing (Bjork, 1975; Craik \& Lockhart, 1972). Elaborative rehearsal during encoding is thought to support the formation of interitem associations and the encoding of item features that are most effective for later remembering (Morris, Bransford, \& Franks, 1977). Critically, given these data, the initial observations relating rote rehearsal to subsequent memorability were reinterpreted as deriving from greater elaboration rather than mere rote maintenance. Consistent with this possibility, evidence for reliance on incidental organizational processes was observed in the rehearsal patterns of subjects even when they were instructed simply to engage in rote maintenance (Rundus, 1971; Tulving, 1966).

At present, a prevalent view expressed by leading theorists is that engagement of phonological rehearsal does not modulate subsequent long-term memorability (Anderson, 2000; Baddeley, 1998). For example, Bower (2000) recently forwarded this perspective in a review of the history of memory research. Based on the failures to observe a link between rote rehearsal and episodic retrieval, Bower concluded that the "repetitive, going over of verbal items results in very little memory later-that is, 'mindless' rehearsal per se is not sufficient to create durable memories" (p. 22). Importantly, this conclusion appears to be based primarily on studies that have probed recollective memory through free recall (Craik \& Watkins, 1973; Tulving, 1966). Subsequent episodic remembrance, however, may be based on multiple mnemonic processes. Whereas free recall indexes processes supporting the recollection of a past event, recognition tests probe recollective processes as well as item strength or familiarity (Dobbins, Khoe, Yonelinas, \& Kroll, 2000; Jacoby, 1991; Tulving, 1985; Mandler, 1980). Assessment of the impact of rote rehearsal on memory formation through free recall may fail to detect the contributions of phonological rehearsal in incrementing item strength and in enhancing recollection that is triggered by representation of the item at test. Indeed, in contrast to the prevalent view, some behavioral evidence suggests that rote rehearsal may contribute to encoding as such rehearsal appears to yield benefits for later "recognition" performance (Green, 1987; Naveh-Benjamin \& Jonides, 1984; Woodward et al., 1973, although see Craik \& Watkins, 1973).

Given the centrality of understanding the relation between working memory mechanisms and long-term learning, an event-related functional magnetic resonance imaging (fMRI) study was conducted to investigate whether the magnitude of neural activation during engagement in rote rehearsal is correlated with or predictive of subsequent memory. Prior neuroimaging studies in humans suggest that the posterior extent of the left inferior prefrontal cortex (pLIPC), the supplementary motor area (SMA), bilateral posterior parietal, and lateral cerebellar regions are components of an integrated network that supports phonological working memory (Jonides et al., 1998). These results are broadly consistent with neurophysiological studies in nonhuman primates that implicate prefrontal- posterior cortical circuits in keeping information in mind (Miller \& Cohen, 2001; Chafee \& Goldman-Rakic, 1998; Fuster, Bauer, \& Jervey, 1985). Here, fMRI was combined with a subsequent memory paradigm (Wagner, Koutstaal, \& Schacter, 1999; Fabiani \& Donchin, 1995; Rugg, 1995; Halgren \& Smith, 1987; Paller, Kutas, \& Mayes, 1987; Sandquist, Rohrbaugh, Syndulko, \& Lindsley, 1980) to determine whether, during rote rehearsal of words, the magnitude of activation in neural regions associated with phonological working memory is correlated with subsequent recognition memory for the words.

During rote rehearsal trials, subjects were scanned while they covertly rehearsed triplets of words that were simultaneously presented in a column and then removed for the remainder of the trial (Figure 1). After scanning, memory performance was assessed using a yes- no item recognition test. Based on these subsequent recognition responses, the fMRI data collected during rote rehearsal were sorted into trials that were later better remembered and those that were later less well remembered. The relation between subsequent memory and activation in neural regions previously associated with phonological working memory (i.e., pLIPC, SMA, bilateral posterior parietal, and lateral cerebellar regions) was then considered. To the extent that phonological working memory processes contribute to

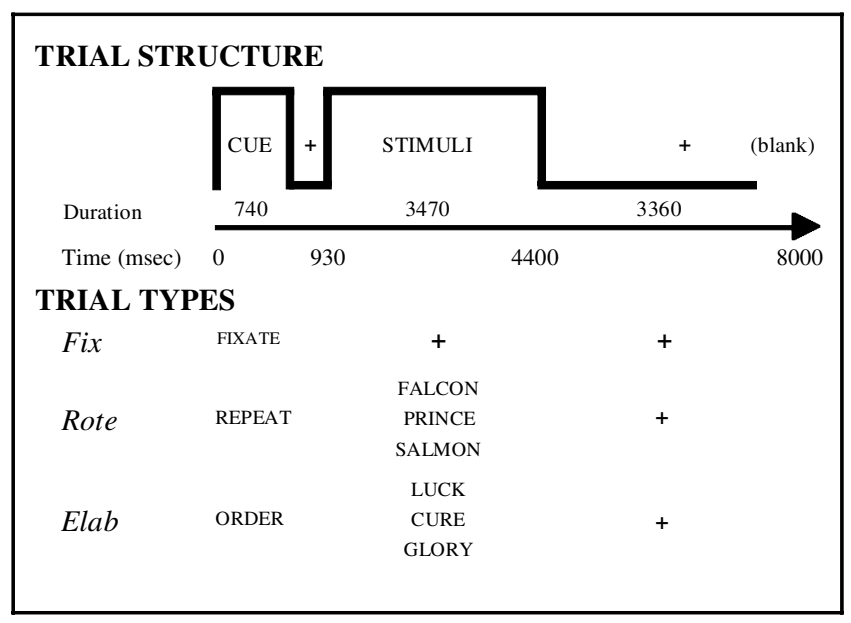

Figure 1. The three trial types (Fix, Rote, and Elab) are illustrated with the appropriate cues and example triplets. Duration of each component of a trial and cumulative trial time is noted by timeline. All experimental trials were $8 \mathrm{sec}$, while the duration of Fixation trials varied. 
encoding, it was predicted that the magnitude of neural activation in these regions would be correlated with subsequent memory performance.

To analyze the extent of semantic processing during performance of the rote rehearsal task, on separate trials subjects also performed an elaborative rehearsal task designed to identify neural regions that mediate semantic elaboration. Subjects were instructed to reorder the words from least to most desirable, again after they had been simultaneously presented and then removed for the remainder of the trial (Figure 1). Activation in these regions was then interrogated for subsequent memory effects during the rote rehearsal task to explore whether trial-by-trial variation in controlled semantic processing during the rote rehearsal task was also correlated with subsequent memory. If regions activated by performance of the elaborative rehearsal task are predictive of subsequent memory for items that were rote-rehearsed, this would provide further evidence that such elaboration impacts encoding and subsequent memorability.

\section{RESULTS}

\section{Subsequent Recognition Memory}

All behavioral effects were significant at an alpha level of .001 , unless otherwise noted. Performance on the item recognition test was considered both collapsed across confidence and segregated by confidence. Analysis of variance (ANOVA) revealed that the probability of responding "old" differed across trial types (Elab, Rote, and New) both when considering overall performance $[F(2,30)=77.03]$ and when restricting attention to high confidence responses $[F(2,30)=72.62]$. Recognition was superior following Elab relative to Rote rehearsal [overall, $F(1,15)=57.82$; high confidence, $F(1,15)=$ 67.35], with the hit rate for the Rote task being superior to the false alarm rate [overall, $F(1,15)=108.10$; high confidence, $F(1,15)=89.29$ ]. The probabilities that an item was endorsed as "old" for Elab, Rote, and New items were $.67, .40$, and .24 (overall), and $.47, .20$, and .08 (high confidence), respectively.

The response latencies during retrieval (Table 1) differed across item type [Elab/Rote/New; $F(2,30)=$ 11.14], with pairwise comparisons revealing that response latencies to New items was significantly longer than those to Rote or Elab studied items, with the studied conditions not differing. Latencies also differed across response type ["High confidence old"/"Low confidence old"/"New"; $F(2,30)=28.35$ ]; latencies for "Low confidence old" responses were significantly longer than those for "New" or "High confidence old" responses, with "New" responses not differing from "High confidence old" responses. Finally, there was a significant interaction between item type and response type $[F(4,60)=4.61, p<.003]$.
Table 1. Response Latencies (and One Standard Error of the Mean) during Subsequent Episodic Recognition

\begin{tabular}{llcc}
\hline & \multicolumn{3}{c}{ Response (msec) } \\
\cline { 2 - 4 } Item Type & "Old-HC" & "Old-LC" & "New" \\
\hline Rote & $1212(67)$ & $2018(187)$ & $1291(77)$ \\
Elab & $1222(97)$ & $1887(158)$ & $1312(82)$ \\
New & $1645(200)$ & $2149(198)$ & $1270(65)$ \\
\hline
\end{tabular}

$\mathrm{HC}=$ high confidence; $\mathrm{LC}=$ low confidence.

Finally, to explore recognition by triplet, triplets were classified according to the number of items later remembered (zero, one, two, or three; collapsed across confidence). Analyses revealed a significant Task $x$ Memory interaction $[F(3,45)=27.33]$. Subjects were more likely to remember all three items from a triplet following Elab relative to Rote rehearsal $[F(1,15)=$ 53.13]. Conversely, subjects were more likely to remember zero or one of the items following Rote relative to Elab rehearsal $\left[F^{\prime} s(1,15)>13.62\right.$; Figure 2$]$. These data replicate the well-established result that subsequent memory is superior following elaborative relative to rote rehearsal (Craik \& Lockhart, 1972).

\section{fMRI Task Effects}

The Rote and the Elab tasks were expected to require phonological maintenance, with phonological working memory demands perhaps being greater during the Elab task (due to the additional phonological operations that are associated with semantic retrieval, and the additional load likely associated with reordering the contents of working memory). Initial fMRI analyses assessed whether the neural regions previously associated with phonological working memory were engaged during performance of the rehearsal tasks (collapsed across task) relative to baseline. Although this contrast does not isolate only those regions associated with phonological rehearsal, nevertheless, this contrast served to determine whether the neural regions that previously have been associated with phonological working memory were active across our two tasks.

Voxel-based statistical analyses revealed that performance of the rehearsal tasks (collapsed across task) elicited activation in visual, parietal, medial temporal, cerebellar, and frontal regions. With respect to the neural regions previously associated with phonological working memory, the experimental tasks significantly engaged the pLIPC ( $\sim$ Brodmann's area [BA 44/6]; region "a" in Figure 3), left cerebellar cortex, the SMA ( $\sim \mathrm{BA} 6)$, and bilateral superior parietal cortex ( $\sim \mathrm{BA} 7 / 40)$. The superior parietal regions converge with prior studies of phonological rehearsal, but are distinct from a more inferior parietal region that also has been associated with phonological working 


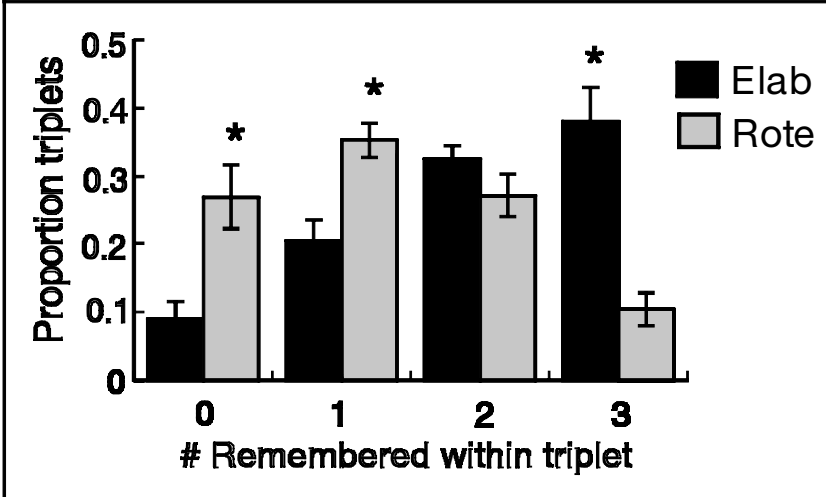

Figure 2. Proportion of triplets from which zero, one, two, or three items were subsequently remembered (collapsed across confidence) from the Elab and Rote conditions (*significant at $p<.001$ ).

memory (Becker, MacAndrew, \& Fiez, 1999). Separate contrasts of each experimental task to baseline revealed that each of the regions associated with phonological working memory was engaged during both of our tasks (Figures 3 and 4; Table 2). ${ }^{1}$ Subsequent voxel-based and region-of-interest (ROI) analyses indicated that the magnitude of responses in these regions were greater during Elab relative to Rote rehearsal [Figure 4A-E; all F's $(1,15)$ $>6.63$, p's $<.03]$.

In addition to engagement, during Rote and Elab trials, of regions previously associated with phonological rehearsal, analyses also indexed prefrontal regions associated with the semantic elaboration and executive control processes required during the Elab task. Consistent with an earlier study (Wagner, Maril, Bjork, \& Schacter, in press), voxel-based analyses revealed that Elab trials additionally elicited activation both in the right dorsolateral prefrontal cortex (DLPFC; $\sim \mathrm{BA} 46 / 9$ ) and in the anterior extent of the left inferior prefrontal cortex (aLIPC; $\sim \mathrm{BA}$ 45/47) when compared to fixation and to Rote trials (see "b" and "c," respectively, in Figure 3). DLPFC and aLIPC did not display above baseline activation during Rote trials (Figures 3 and 4). DLPFC previously has been implicated in the engagement of executive control mechanisms that permit comparisons across, manipulation of, and selection from among representations being maintained in working memory (e.g., Wagner et al., in press; Rowe, Toni, Josephs, Frackowiak, \& Passingham, 2000; D’Esposito, Postle, Ballard, \& Lease, 1999; Petrides, 1996). The aLIPC region previously has been implicated in the controlled retrieval of long-term semantic knowledge (e.g., Wagner, Koutstaal, Maril, Schacter, \& Buckner, 2000; Wagner, Paré-Blagoev, Clark, \& Poldrack, 2001; Poldrack et al., 1999; Price, Moore, Humphreys, \& Wise, 1997).

\section{fMRI Subsequent Memory Effects}

The central question of interest was whether rehearsalrelated activity correlated with later memory perform- ance. Subjects who had fewer than 15 trials within a cell were not included in this analysis (Rote, four subjects excluded; Elab, three subjects excluded). Critically, for trials that were rote-rehearsed, ROI-based analyses revealed that the magnitude of activation in each of the four regions previously associated with the maintenance of phonological representations was predictive of later memory performance (Figure 4A-E). Specifically, each ROI demonstrated a Memory (zero, one, or two items remembered) $\times$ Time $(0-20 \mathrm{sec})$ interaction [all $F^{\prime}$ s $(20,220)>1.92$, p's < .02; except SMA, $F(20,220)=$ $1.55, p=.06]$. Planned contrasts revealed that the peak response in the pLIPC, bilateral superior parietal, SMA, and left cerebellum was greater for trials from which two items were later remembered versus trials from which zero or one item was later remembered [all $F$ 's $(1,11)>$ 7.20, p's < .01; except SMA, F's $(1,11)>3.74$, p's $=.05$ ] Thus, greater activation in each of these regions was correlated with superior subsequent memory.

For these same regions, although activity was greater during Elab than during Rote rehearsal (Figures 3 and 4), trial-by-trial differences in the magnitude of activity during Elab trials was not predictive of later memory $\left[F^{\prime} s(20,240)<1.48, p^{\prime}\right.$ 's $>.09$; except right superior parietal, $F(20,240)=1.74, p<.03$, however, no significant difference was observed when considering the peak response]. The only region that predicted subsequent memory for the Elab trials was the left hippocampus, and this effect will be considered more fully in a subsequent publication. The relative absence of subsequent memory effects for the Elab trials is surprising given that numerous prior studies have observed subsequent memory effects in the frontal and temporal regions following the semantic encoding of single words (e.g., Baker, Sanders, Maccotta, \&

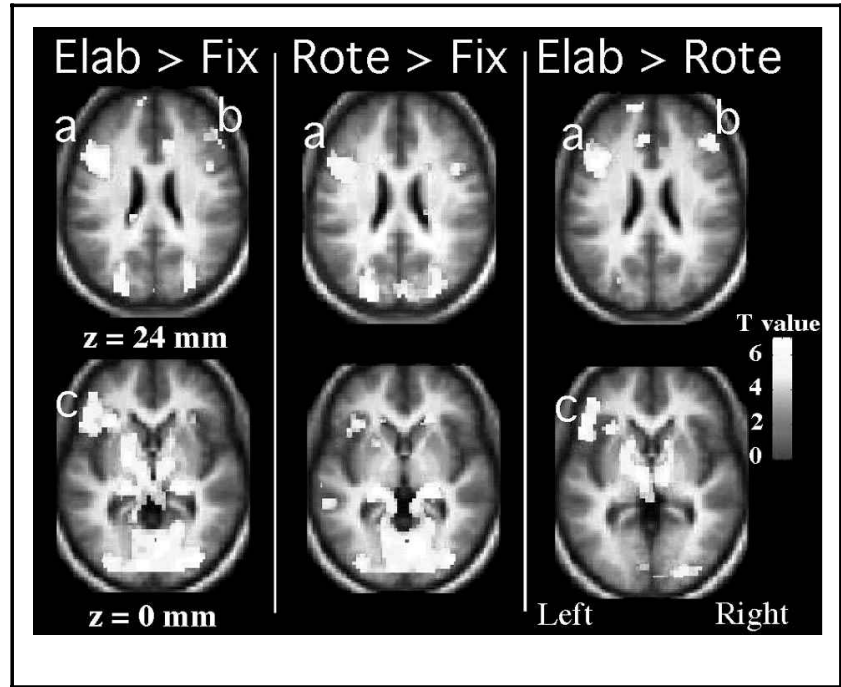

Figure 3. Rote and Elab rehearsal jointly elicited activation in the pLIPC (region a), whereas Elab rehearsal differentially engaged the right DLPFC (b) and aLIPC (c). Distance from the anterior-posterior commissure plane is listed in millimeters. 


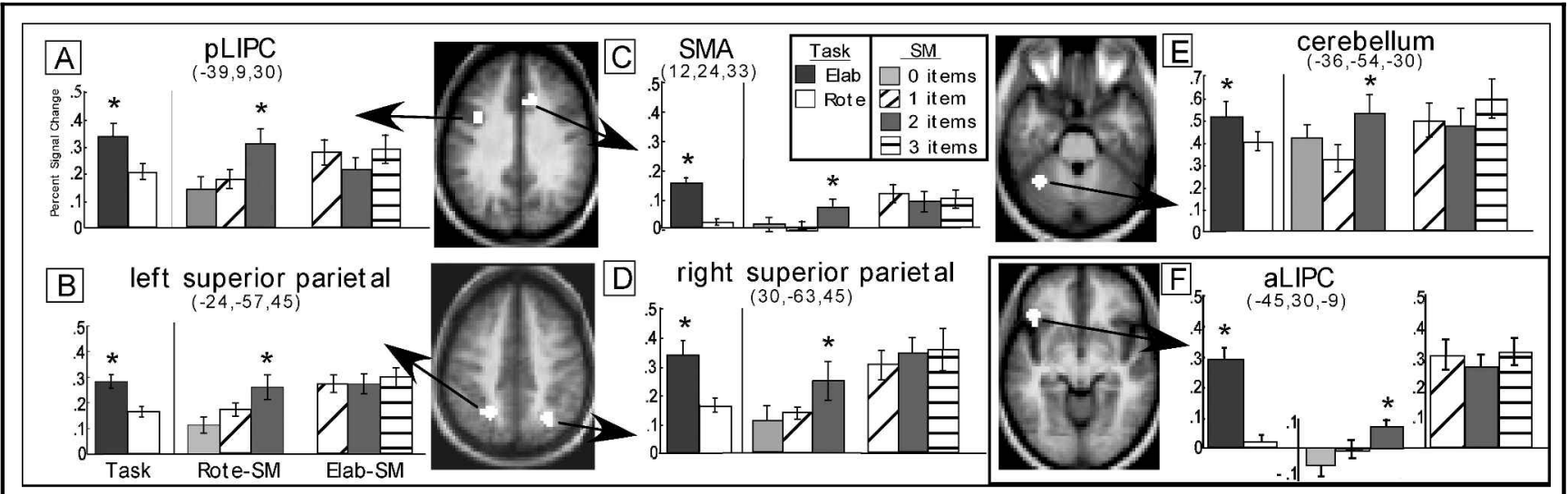

Figure 4. Subsequent memory effects for the Rote condition were observed in neural regions previously associated with phonological rehearsal (pLIPC, SMA, left and right superior parietal, and left cerebellum) as well as in the aLIPC region associated with semantic elaboration. Displayed are the peak responses for both tasks (Elab vs. Rote) and for trials sorted by whether zero, one, or two items from the Rote trials and one, two, or three items from the Elab trials were later remembered (*significant effects).

Buckner, 2001; Otten, Henson, \& Rugg, 2001; Kirchhoff, Wagner, Maril, \& Stern, 2000; Henson, Rugg, Shallice, Josephs, \& Dolan, 1999; Wagner et al., 1998). It is unclear why the present Elab task did not yield similar results. One possibility is that, because subjects were required to semantically process three words per trial (compared to one in all prior studies), the BOLD response during these trials may have been saturated, thus masking any differences that might have been correlated with subsequent memory. Irrespective of the reason for this null result, the absence of such subsequent memory effects in the Elab condition should be interpreted cautiously given the presence of such effects in the literature.

To assess whether semantic and high-level control processes were also correlated with subsequent memory following rote rehearsal, ROI analyses were conducted on the ALIPC and DLPFC regions observed in the Elab > baseline contrast. These analyses revealed that the magnitude of activity in the aLIPC was predictive of later memory following Rote rehearsal [Memory $\times$ Time, $F(20,220)=1.59, p=.05$; peak response, $F(1,11)=$ $4.14, p<.05$ ] but not following Elab rehearsal $(F<1.0)$ (Figure $4 \mathrm{~F}$ ). In contrast, activity in the right DLPFC did not show a significant subsequent memory effect for either Rote $[F(20,220)=1.15, p=.29]$ or Elab $(F<1.0)$ trials.

Finally, the subsequent memory analyses also revealed an intriguing pattern when considering the task effects. In particular, as previously mentioned, when collapsing across subsequent memory performance, the Elab task elicited greater activation in the pLIPC, bilateral parietal, SMA, and cerebellum relative to the Rote task. However, consideration of the Rote trials that were later well remembered revealed that the magnitude of activation in these regions was similar to that observed during the Elab trials (irrespective of subse- quent memory level). Although one might be tempted to conclude that this activation during the later well remembered Rote trials reflects performance of the Elab task during these trials, the data from the aLIPC region would suggest otherwise. That is, in contrast to the four regions previously associated with phonological working memory, the magnitude of activation during the later well remembered Rote trials in the aLIPC-a region associated with semantic elaboration-was still markedly below that in the Elab trials. Thus, we suggest that this pattern is consistent with the robust recruitment of neural computations associated with phonological rehearsal during the later well-remembered Rote trials, and that this recruitment facilitated episodic encoding and later remembering.

\section{DISCUSSION}

The central aim of the present study was to determine whether differential engagement of rote rehearsal mechanisms-as indexed by activation in neural correlates of phonological working memory-is associated with differences in later long-term retrieval. The results revealed three important outcomes. First, regions previously associated with phonological rehearsal (i.e., pLIPC, bilateral parietal, SMA, and cerebellum) were activated during both rote item rehearsal and elaborative rehearsal. This outcome demonstrates that the maintenance of verbal codes, in the absence of a decision or probe phase, elicits activation in these components of the phonological working memory system. Second, the subsequent memory analysis revealed that the magnitude of activity elicited in each region previously associated with phonological rehearsal was predictive of later memory for words that were rote-rehearsed. Importantly, in contrast to the prevalent view of the impact of rote rehearsal on long-term memory, these data 
Table 2. Regions That Exhibited Significant Activation in the Rote Task Relative to Fixation

\begin{tabular}{|c|c|c|c|c|c|}
\hline \multirow[b]{2}{*}{ Region } & \multicolumn{3}{|c|}{ MNI Coordinates } & \multirow{2}{*}{$\begin{array}{l}\sim \text { Brodmann's } \\
\text { Areas }\end{array}$} & \multirow{2}{*}{$\begin{array}{c}\text { Peak Z Score } \\
\text { (No. Voxels) }\end{array}$} \\
\hline & $x$ & $y$ & $z$ & & \\
\hline R. mid. occipital gyrus & 33 & -87 & 12 & 19 & $6.32(5,169)$ \\
\hline R. pulvinar & 21 & -30 & -3 & & 5.78 \\
\hline L. mid. occipital gyrus & -27 & -93 & 12 & 19 & 5.76 \\
\hline L. intraparietal sulcus & -24 & -60 & 45 & $40 / 7$ & 5.01 \\
\hline \multirow[t]{2}{*}{ R. intraparietal sulcus } & 30 & -60 & 45 & $40 / 7$ & 4.57 \\
\hline & 27 & 60 & 51 & $40 / 7$ & 4.52 \\
\hline R. precentral gyrus & -45 & 0 & 48 & 6 & $5.10(651)$ \\
\hline L. inferior frontal gyrus & -45 & 18 & 24 & $44 / 6$ & 4.59 \\
\hline L. precentral gyrus & -54 & -6 & 45 & 6 & 4.55 \\
\hline \multirow[t]{3}{*}{ L. superior frontal gyrus } & -3 & 6 & 60 & 6 & $5.00(208)$ \\
\hline & -9 & 15 & 45 & 6 & 4.98 \\
\hline & -15 & 18 & 24 & 6 & 4.49 \\
\hline R. insula & 36 & 21 & -6 & $44 / 45$ & $4.94(25)$ \\
\hline \multirow[t]{2}{*}{ R. precentral gyrus } & 45 & 12 & 21 & 6 & $4.52(57)$ \\
\hline & 51 & 9 & 33 & 6 & 3.76 \\
\hline R. caudate & 21 & 30 & 0 & & $4.37(32)$ \\
\hline \multirow[t]{3}{*}{ L. insula } & -36 & 24 & -3 & $44 / 45$ & $4.34(66)$ \\
\hline & -33 & 24 & 12 & $44 / 45$ & 3.96 \\
\hline & -45 & 21 & 0 & $44 / 45$ & 3.54 \\
\hline L. cerebellum & -18 & -30 & -30 & & $4.19(7)$ \\
\hline L. superior temporal sulcus & -66 & -24 & -3 & $21 / 22$ & $4.07(6)$ \\
\hline \multirow[t]{2}{*}{ R. precentral gyrus } & 48 & -3 & 54 & 6 & $3.95(8)$ \\
\hline & 42 & -6 & 60 & 6 & 3.50 \\
\hline R. superior frontal sulcus & 27 & 3 & 48 & 6 & $4.99(12)$ \\
\hline R. occipito-temporal sulcus & 42 & -30 & -21 & 20 & $3.74(6)$ \\
\hline Pons & 0 & -27 & 66 & & $3.69(23)$ \\
\hline \multirow[t]{2}{*}{ L. superior temporal sulcus } & -60 & -39 & 0 & $21 / 22$ & $3.67(38)$ \\
\hline & -48 & -39 & 3 & $21 / 22$ & 3.54 \\
\hline \multirow[t]{2}{*}{ L. putamen } & -21 & 9 & 3 & & $3.62(13)$ \\
\hline & -24 & 15 & -3 & & 3.34 \\
\hline R. precentral gyrus & 57 & 3 & 42 & 6 & $3.62(5)$ \\
\hline L. caudate & -18 & 30 & 0 & & $3.52(6)$ \\
\hline R. cingulate sulcus & 9 & 24 & 36 & 32 & $3.49(7)$ \\
\hline R. cerebellum & 9 & -66 & -36 & & $3.40(5)$ \\
\hline \multirow[t]{2}{*}{ R. superior frontal sulcus } & 33 & -18 & 51 & 6 & $3.35(15)$ \\
\hline & 39 & -18 & 57 & 6 & 3.28 \\
\hline
\end{tabular}

L. = left; R. = right; mid. = middle. 
suggest that increased engagement of regions thought to be involved in phonological rehearsal mechanisms leads to better subsequent memory and thus, presumably, more effective encoding. Finally, while the aLIPC, a region previously shown to mediate controlled retrieval of semantic codes (Wagner et al., 2001; Poldrack et al., 1999) was not engaged-on average-during rote rehearsal (Figures 3 and 4), the magnitude of activity in this region was nevertheless predictive of later memory following rote rehearsal. These data suggest that in addition to the contributions of phonological working memory to later remembering, when semantic elaboration is engaged-even when not required for task performance-subsequent memory is enhanced (Wagner et al., 1998; Craik \& Lockhart, 1972).

Prior functional imaging studies have implicated the pLIPC, bilateral parietal, SMA, and cerebellar regions in phonological working memory (Jonides et al., 1998). The logic of the present study was to capitalize on the prior association between these regions and phonological maintenance processes so as to address a fundamental question regarding the consequences of recruiting a set of cognitive operations (phonological rehearsal) for long-term memory. Thus, the goal was to rely on the extant functional imaging literature regarding structure-function relations so as to draw inferences at the cognitive level based on differential functional signals (e.g., Thompson-Schill, Aguirre, D'Esposito, \& Farah, 1999). It is impossible, of course, to be absolutely certain that activation in these four regions during performance of a rote rehearsal task, such as that in the present study, unambiguously reflects the engagement of phonological working memory mechanisms. Nevertheless, to the extent that activation in these regions reflects processes that contribute to phonological working memory-and the extant literature would suggest that this is a reasonable assumption-then we may draw inferences about the impact of differential recruitment of these processes on episodic encoding (as indexed by subsequent retrieval).

The present data provide clear evidence that engagement of the pLIPC, bilateral parietal, SMA, and cerebellar regions during rote item maintenance influences later memorability for the items. To our knowledge, this is the first demonstration that activity in regions previously shown to be engaged during the maintenance of phonological codes, as determined by neuropsychological and neuroimaging studies (Smith \& Jonides, 1998; Awh et al., 1996; Jonides et al., 1996; Paulesu, Frith, \& Frackowiak, 1993; Baddeley, Lewis, \& Vallar, 1984; Basso, Spinnler, Vallar, \& Zanobio, 1982), predict later explicit memory for items that were roterehearsed. Prior event-related fMRI investigations of episodic encoding have indexed the neural regions that predict subsequent memory following either semantic encoding (e.g., deciding if a word is abstract or concrete; Wagner et al., 1998) or nonsemantic encoding (e.g., deciding if the first and last letters of a word are in ascending or descending alphabetical order; Otten et al., 2001). In contrast to the present results, while these studies have observed subsequent memory effects in some of the four regions associated with phonological rehearsal (most notably in the pLIPC; Baker et al., 2001; Otten et al., 2001; Wagner et al., 1998), in no prior study were all four regions observed to predict later remembering. This difference between the present results and the prior literature likely reflects the fact that the present experiment is the first to explore subsequent memory effects following encoding via a task that demands extensive rote rehearsal. In particular, the present rote rehearsal condition required subjects to access the phonological codes associated with a set of visually presented words and to then rehearse these codes for a period of seconds. The data indicate that the mechanisms that support such phonological access and rehearsal appear to contribute to long-term memory formation, and stand in contrast to the commonly held perspective that rote rehearsal of information does not guide long-term memory formation (Anderson, 2000; Bower, 2000; Baddeley, 1998; Craik \& Lockhart, 1972).

Event-by-event differences in activation associated with phonological working memory during rote rehearsal may derive from a number of possible mechanisms. First, trials eliciting greater activation may constitute events for which there was a greater extent of rehearsal during encoding. For example, it is possible that the items in triplets that were subsequently better remembered received more rehearsals than did the items in triplets that were subsequently less well remembered. Prior behavioral data suggest that the greater the number of actual rehearsal repetitions an item receives, the more likely it will be remembered (Rundus, 1971), at least for the first few repetitions (Naveh-Benjamin \& Jonides, 1984). Alternatively, the differential engagement of neural regions associated with phonological rehearsal may reflect Subject $\times$ Item interactions. Recent fMRI data from our laboratory (Clark \& Wagner, unpublished observations), in conjunction with neuropsychological and cognitive behavioral findings (Baddeley, Gathercole, \& Papagno, 1998), suggest that phonological control processes may be particularly important for the assembly and encoding of unfamiliar word forms. An item analysis of the present data indicated that the observed subsequent memory effects do not reflect item confounds, as different items were remembered by different subjects. Nevertheless, it remains possible that the items that were more likely to be remembered by a particular subject were items that were less phonologically familiar to that subject. Were this the case, these items would have placed a greater demand on phonological control processes, as suggested by the greater activation, and this increased demand may have contributed to the enhanced encoding of these items. Further efforts should serve to 
specify the source of the observed trial-by-trial differences. Irrespective of the source of these differences, the present data suggest that phonological working memory processes that support phonological access and maintenance contribute to episodic encoding such that an item can be subsequently recognized following a delay of (on average) about $45 \mathrm{~min}$.

In the present rote rehearsal paradigm, learning was incidental, and thus likely did not motivate a strategy of intentional elaboration on the meaning of the items that were to be rote-rehearsed. That is, subjects were likely to have engaged in minimal systematic access and evaluation of semantic information during the rote trials, with controlled semantic processing in this condition being markedly less than that during elaborative trials. In accord with this perspective, on average, the aLIPCwhich has been associated with controlled, rather than automatic, semantic retrieval and evaluation (Wagner et al., 2001; Poldrack et al., 1999; Price et al., 1997)— was not engaged during the rote rehearsal condition (Figures 3 and 4). Nevertheless, the observation that the magnitude of aLIPC activation also predicted subsequent memory for rote-rehearsed items raises the possibility that event-by-event variations in semantic elaboration, and not phonological rehearsal per se, may be solely responsible for the enhanced subsequent memory performance. From this perspective, the variations in activation of the regions associated with phonological rehearsal that were shown to predict later memory might merely reflect differential demands for maintaining the newly retrieved semantic information, and is thus a "by-product" of bringing on-line such semantic codes. While it is clear that semantic elaboration and aLIPC computations do impact subsequent memory (Kirchhoff et al., 2000; Henson et al., 1999; Wagner et al., 1998; Craik \& Lockhart, 1972), the present data diverge from earlier reports and thus suggest that rote rehearsal of the items themselves impacts later remembering. In particular, all prior investigations of the neural correlates of subsequent memory have failed to reveal predictive activation in each of the four neural regions previously associated with phonological working memory (Baker et al., 2001; Otten et al., 2001; Kirchhoff et al., 2000; Henson et al., 1999; Wagner et al., 1998). Importantly, this was the case even in studies that explored subsequent memory following semantic elaboration (Baker et al., 2001; Otten et al., 2001; Kirchhoff et al., 2000; Henson et al., 1999; Wagner et al., 1998). To the extent that the present subsequent memory effects in the pLIPC, bilateral parietal, SMA, and cerebellar regions reflect second-order effects following semantic elaboration, then earlier studies that directly elicited semantic elaboration through task instructions should have yielded a similar pattern of subsequent memory effects in all of these regions. Again, this was not the case. Thus, the present study suggests that during rote rehearsal both phonological working memory and semantic elaboration mechanisms contribute to episodic memory formation.

The present data suggest that differential recruitment of the neural underpinnings of phonological rehearsal appears to yield superior recognition for the rehearsed items. A central question that awaits further investigation is whether rote rehearsal selectively increments item strength or whether such rehearsal also impacts the formation of representations that support subsequent recollection. It has been suggested that maintenance rehearsal on its own does not lead to the formation of interitem associations that facilitate recall, but rather, might specifically increase item familiarity processes that are sufficient to guide recognition but not recollection (Nairne, 1986; Gillund \& Shiffrin, 1984; Geiselman \& Bjork, 1980). Along these lines, behavioral evidence indicates that increased engagement in rote rehearsal serves to increase "knowing" but not "remembering" (Gardiner, Gawlik, \& Richardson-Klavehn, 1994). Interestingly, in the present study, rote-rehearsed trials that were later better remembered (i.e., subsequently recognized two of the words) resulted in higherconfidence remembering (ratio of items remembered with high-to-low confidence was 1:1.3) relative to trials that were less well remembered (i.e., recognized one of the words; ratio of 1:2.3). Although these ratios might suggest that better subsequent memory was associated with increased recollection, behavioral data indicate that retrieval confidence may not directly map to recollection versus familiarity (Gardiner \& Java, 1990). Thus the present data do not resolve whether differential activation, during rote rehearsal, of regions associated with phonological maintenance specifically increases item familiarity without facilitating the formation of representations that support recollection.

Collectively, the present findings represent the first functional neurobiological evidence suggesting that cognitive control processes that subserve rote maintenance of information in a transient form also contribute to the formation of more durable memories. Such maintenance processes may influence the likelihood or extent to which item information is passed onto the medial temporal lobe memory system, a region known to be critical in episodic memory formation (Squire, 1992). Future studies should serve to further clarify how merely keeping an experience in mind for a few seconds influences one's ability to later bring the experience to mind.

\section{METHODS}

\section{Participants}

Participants were 16 right-handed, native speakers of English (eight women; ages 18- 35 years), with normal or corrected-to-normal vision. Participants received a US\$50 remuneration. Informed consent was obtained in a manner approved by the Human Studies Committee 
of the MGH and the Committee on the Use of Humans as Experimental Subjects at MIT.

\section{Stimuli and Behavioral Procedures}

Stimuli consisted of 224 visually presented triplets of nouns printed in uppercase letters and in a column. For counterbalancing purposes, sets of 14 triplets were formed such that the sets were matched for mean word length, word frequency, and "desirability" (normative desirability was indexed by collecting desirability ratings from an independent sample of participants). Across subjects, stimuli were counterbalanced such that each set of stimuli served equally often in the two experimental conditions - rote and elaborative rehearsaland across the eight runs of the scan session.

Prior to fMRI scanning, participants received extensive practice on the experimental tasks, both outside and inside the magnet, so as to ensure that they understood the instructions and could perform the tasks in the time allotted. Over the course of eight event-related fMRI scans, 112 eight-second trials from each of two trial types (Figure 1) were intermixed with variable-duration visual fixation null events. The order of the conditions (rote and elaborative) within each scan was determined using an optimal sequencing program designed to maximize the efficiency of recovery of the BOLD response, based on the assumption of a linear time invariant system (Dale, 1999; Dale \& Buckner, 1997). The periods of visual fixation lasted between 2 and $22 \mathrm{sec}$, "jittered" in increments of $2 \mathrm{sec}$, as determined by the optimization algorithm. During fixation null events (Fix), the cue "FIXATE" indicated that subjects should fixate on a "+" sign throughout the duration of its appearance on the screen.

During rote rehearsal trials (Rote), the cue "REPEAT" indicated that subjects should covertly rehearse the word triplet in the order presented throughout the duration of the trial. Three words were presented simultaneously in a column and then removed from the screen for the remainder of the trial (Figure 1). Importantly, and in contrast to many investigations of working memory, there was no probe or decision phase at the end of the trial; that is, participants were not required to compare a test probe against the contents of working memory. Thus, this task primarily necessitated recruitment of phonological access and rote maintenance processes, and placed minimal demands on comparison across or selection from among representations within working memory.

An elaborative rehearsal task (Elab) was included to identify the neural correlates of controlled semantic processing and of "higher-level" cognitive control processes that permit selection of, or comparisons across, representations within working memory. Specification of the neural substrates of such control processes was designed to permit targeted ROI assessment so as to determine the extent of engagement of these processes during rote rehearsal and to determine their contributions to subsequent explicit remembering. With these goals in mind, during Elab trials, the cue "ORDER" indicated that subjects should covertly reorder the words in the triplet along the semantic dimension of subjective "desirability," going from least to most desirable, again for the duration of the trial. The instructions emphasized that subjects should settle on their order only after considering the desirability of each item in relation to the other items in the triplet. As discussed previously, Elab trials were expected to rely on phonological access and rote maintenance mechanisms as much as or perhaps more so than in the Rote trials. In contrast to Rote rehearsal, the Elab condition also required semantic processing and higher-level cognitive control.

To assess the extent to which rote and elaborative rehearsal contributes to memory formation, memory for the items encountered during the Rote and Elab trials was evaluated using an item-based recognition memory test administered approximately $20 \mathrm{~min}$ after the last fMRI scan. During test, all previously encountered words and a set of unstudied distractors (i.e., New items) were presented individually. Subjects indicated whether they remembered having studied the item, further designating their confidence ("high" or "low") when responding "studied." These behavioral measures of subsequent remembering were used to conduct a subsequent memory analysis of the fMRI data.

\section{fMRI Procedures}

Scanning was performed on a 1.5-T Siemens Sonata MRI system using a whole-head coil. Functional data were acquired using a gradient-echo echo-planar pulse sequence $(\mathrm{TR}=2 \mathrm{sec}, \mathrm{TE}=40 \mathrm{msec}, 21$ axial slices, $3.125 \times 3.125 \times 5 \mathrm{~mm}, 1 \mathrm{~mm}$ interslice gap, 168 volume acquisitions per run). High-resolution T1weighted (MP-RAGE) anatomical images were collected for anatomical visualization. Head motion was restricted using a bite-bar apparatus. Visual stimuli were projected via a collimating lens onto a screen that was viewed through a mirror.

Data were preprocessed using SPM99 (Wellcome Department of Cognitive Neurology, London). Images were corrected for differences in slice acquisition timing by resampling all slices in time to match the first slice, followed by motion correction across all runs (using sinc interpolation). Structural and functional data were spatially normalized to an EPI template based on the MNI305 stereotactic space (Cocosco, Kollokian, Kwan, \& Evans, 1997)—an approximation of Talairach space (Talairach \& Tournoux, 1988) — using a 12-parameter affine transformation along with a nonlinear transformation using cosine basis functions. Images were resampled into 3-mm cubic voxels and then 
spatially smoothed with an 8-mm FWHM isotropic Gaussian kernel.

Statistical analysis was performed using the general linear model in SPM99. Trials from each condition were modeled using a canonical hemodynamic response function and its temporal derivative (in a separate analysis, trials were modeled as 8-sec epochs; results were comparable to the canonical HRF model and thus we report the outcome of the latter). Effects were estimated using a subject-specific fixed-effects model, with session-specific effects and low-frequency signal components treated as confounds. Linear contrasts were used to obtain subject-specific estimates for each effect. To assess the effect of rehearsal, the experimental trials (collapsed across task) were contrasted to baseline (Fix). This contrast was expected to yield increased activation in regions supporting rote phonological rehearsal as both tasks demanded phonological maintenance. To assess the differential response during the two rehearsal tasks, Rote and Elab trials were directly contrasted. Finally, to assess the response associated with each rehearsal task relative to baseline, each condition (Rote and Elab) was separately contrasted with baseline. Importantly, the contrast of Elab relative to baseline was expected to reveal additional regions associated with semantic elaboration and "higher-level" executive control. The subject-specific estimates derived from each of these contrasts were entered into a second-level group analysis treating subjects as a random effect, using a one-sample $t$ test against a contrast value of zero at each voxel. Regions were considered reliable to the extent that they consisted of at least 5 contiguous voxels that exceeded an uncorrected threshold of $p<.001$.

To further explore the correlates of rote and elaborative rehearsal, ROI analyses were performed. Spherical ROIs included all significant voxels within a 6-mm radius of each chosen maximum identified in the group statistical map. Signal within each ROI was calculated for each individual subject by selectively averaging the data with respect to peristimulus time for trials in each condition. The resultant hemodynamic response associated with each trial type reflects percent signal change relative to the fixation baseline from 0 to 20 sec peristimulus time. These data were then subjected to mixed-effects ANOVA that treated task (Rote/Elab) and time $(0-20 \mathrm{sec})$ as repeated measures and subjects as a random effect.

ROIs were functionally defined in two ways. First, we identified regions demonstrating a greater response during the rehearsal trials (collapsed across task) relative to baseline. These regions are unbiased with respect to the Rote/Elab manipulation and provide leverage for ROI-based assessment of the effect of task and of subsequent memory in the neural regions associated with phonological working memory. In addition, regions were defined based on the contrast of Elab relative to baseline, as this contrast was expected to reveal additional regions that were correlated with semantic elaboration and higher-level executive control.

To examine the effect of task, the significance of the Task $\times$ Time interaction was tested for each ROI. For regions demonstrating a significant interaction, an effect of task was further assessed through planned contrasts that compared the percent signal change associated with the Rote and the Elab trials at the time point corresponding to the peak response (defined from the mean of the two trial types). To examine "within-task" differences in the hemodynamic response correlated with subsequent memory, trials were divided into those in which subjects later remembered zero, one, two, or three items from a triplet. This analysis was conducted collapsing across confidence because there were insufficient trials to permit analysis restricted only to the high confidence and forgotten trials (Wagner et al., 1998). Moreover, owing to the small number of trials in which subjects remembered all three items from a triplet that was rote-rehearsed and zero items from a triplet that was elaboratively rehearsed, these bins were not included in the subsequent memory analysis. For each task (Rote or Elab), the ROI analysis examined whether there was a reliable Memory (zero/one/two or one/two/ three items remembered) $\times$ Time $(0-20 \mathrm{sec})$ interaction, with planned contrasts further exploring whether the peak magnitude of the response differed by subsequent memory. A Huynh- Feldt correction for nonsphericity was implemented for all ROI analyses.

\section{Acknowledgments}

Supported by a Merck/MIT fellowship, the NIDCD (DC04466), Ellison Medical Foundation, Surdna Foundation, and P. Newton. We thank M. Potter and I. Dobbins for insightful comments, and M. Hutson for assistance with data collection.

Reprint requests should be sent to Lila Davachi, Department of Brain and Cognitive Sciences, NE 20-343, MIT, Cambridge, MA 02139 or via e-mail: lila@psyche.mit.edu.

The data reported in this experiment have been deposited in the National fMRI Data Center (http://www.fmridc.org). The accession number is 2-2001-11225.

\section{Note}

1. A complete list of significant activations observed during the Elab > Fix and Elab > Rote contrasts is available upon request from the first author.

\section{REFERENCES}

Anderson, J. R. (2000). Learning and memory: An integrated approach. New York: Wiley.

Atkinson, R. C., \& Shiffrin, R. M. (1968). Human memory: A proposed system and its control processes. In K. W. Spence \& J. T. Spence (Eds.), The psychology of learning and motivation: Advances in research and theory (vol. 2). New York: Academic Press. 
Awh, E., Jonides, J., Smith, E. E., Schumacher, E. H., Koeppe, R. A., \& Katz, S. (1996). Dissociation of storage and rehearsal in verbal working memory: evidence from PET. Psychological Science, 7, 25- 31.

Baddeley, A., Gathercole, S., \& Papagno, C. (1998). The phonological loop as a language learning device. Psychological Review, 105, 158- 173 .

Baddeley, A. D. (1998). Human memory: Theory and practice (Rev. ed.). Needham Heights: Allyn and Bacon.

Baddeley, A. D., Lewis, V., \& Vallar, G. (1984). Exploring the articulatory loop. Quarterly Journal of Experimental Psychology, 36A, 233- 252.

Baker, J. T., Sanders, A. L., Maccotta, L., \& Buckner, R. L. (2001). Neural correlates of verbal memory encoding during semantic and structural processing tasks. NeuroReport, 12, 1251- 1256.

Basso, A., Spinnler, H., Vallar, G., \& Zanobio, M. E. (1982). Left hemispheric damage and selective impairment of auditory short-term memory: A case study. Neuropsychologia, 20, 263- 274

Becker, J. T., MacAndrew, D. K., \& Fiez, J. A. (1999). A comment on the functional localization of the phonological storage subsystem of working memory. Brain and Cognition, 41, 27- 38.

Bjork, R. A. (1975). Short-term storage: The ordered output of a central processor. In F. Restle, R. M. Shiffrin, N. J. Castellan, H. R. Lindeman \& D. B. Pisoni (Eds.), Cognitive theory (vol. 1, pp. 151-171). Hillsdale, NJ: Erlbaum.

Bower, G. H. (2000). A brief history of memory research. In E. Tulving \& F. I. M. Craik (Eds.), The Oxford handbook of memory (pp. 3-32). New York: Oxford University Press.

Chafee, M. V., \& Goldman-Rakic, P. S. (1998). Matching patterns of activity in primate prefrontal area $8 \mathrm{a}$ and parietal area 7ip neurons during a spatial working memory task. Journal of Neurophysiology, 79, 2919- 2940.

Cocosco, C. A., Kollokian, V., Kwan, R. K. S., \& Evans, A. C. (1997). Brainweb: online interface to a 3D MRI simulated brain database. Neuroimage, 5, 425.

Craik, F. I. M., \& Lockhart, R. S. (1972). Levels of processing: A framework for memory research. Journal of Verbal Learning and Verbal Behavior, 11, 671- 684.

Craik, F. I. M., \& Watkins, M. J. (1973). The role of rehearsal in short-term memory. Journal of Verbal Learning and Verbal Behavior, 12, 599- 607.

Dale, A. M. (1999). Optimal experimental design for eventrelated fMRI. Human Brain Mapping, 8, 109-114.

Dale, A. M., \& Buckner, R. L. (1997). Selective averaging of rapidly presented individual trials using fMRI. Human Brain Mapping, 5, 329-340.

D’Esposito, M., Postle, B. R., Ballard, D., \& Lease, J. (1999). Maintenance versus manipulation of information held in working memory: An event-related fMRI study. Brain and Cognition, 41, 66- 86.

Dobbins, I. G., Khoe, W., Yonelinas, A. P., \& Kroll, N. E. A. (2000). Predicting individual false alarm rates and signal detection theory: A role for remembering. Memory and Cognition, 28, 1347-1356.

Ebbinghaus, H. (1885/1964). Memory: A contribution to experimental psychology. New York: Dover.

Fabiani, M., \& Donchin, E. (1995). Encoding processes and memory organization: A model of the von Restorff effect. Journal of Experimental Psychology: Learning, Memory, and Cognition, 21, 224- 240.

Fuster, J. M., Bauer, R. H., \& Jervey, J. P. (1985). Functional interactions between inferotemporal and prefrontal cortex in a cognitive task. Brain Research, 330, $299-307$.

Gardiner, J. M., Gawlik, B., \& Richardson-Klavehn, A. (1994). Maintenance rehearsal affects knowing, not remembering; elaborative rehearsal affects remembering, not knowing. Psychonomic Bulletin and Review, 1, 107-110.

Gardiner, J. M., \& Java, R. I. (1990). Recollective experience in word and nonword recognition. Memory and Cognition, 18, 23-30.

Geiselman, R. E., \& Bjork, R. A. (1980). Primary versus secondary rehearsal in imagined voices: Differential effects on recognition. Cognitive Psychology, 12, 188- 205.

Gillund, G., \& Shiffrin, R. M. (1984). A retrieval model for both recognition and recall. Psychological Review, 91, 1- 67.

Green, R. L. (1987). Effects of maintenance rehearsal on human memory. Psychological Bulletin, 102, 403- 413.

Halgren, E., \& Smith, M. E. (1987). Cognitive evoked potentials as modulatory processes in human memory formation and retrieval. Human Neurobiology, 6, 129- 139.

Henson, R. N., Rugg, M. D., Shallice, T., Josephs, O., \& Dolan, R. J. (1999). Recollection and familiarity in recognition memory: an event-related functional magnetic resonance imaging study. Journal of Neuroscience, 19, 3962- 3972.

Jacoby, L. L. (1991). A process dissociation framework: Separating automatic from intentional uses of memory. Journal of Memory and Language, 30, 513- 541.

Jonides, J., Reuter-Lorenz, P., Smith, E. E., Awh, E., Barnes, L., Drain, M., Glass, L., Lauber, E., Patalano, A., \& Schumacher, E. H. (1996). In D. Medin (Ed.), The psychology of learning and motivation (pp. 43-88). New York: Academic Press.

Jonides, J., Schumacher, E. H., Smith, E. E., Koeppe, R. A., Awh, E., Reuter-Lorenz, P. A., Marshuetz, C., \& Willis, C. R. (1998). The role of parietal cortex in verbal working memory. Journal of Neuroscience, 18, 5026- 5034.

Kirchhoff, B. A., Wagner, A. D., Maril, A., \& Stern, C. E. (2000). Prefrontal- temporal circuitry for novelty encoding and subsequent memory. Journal of Neuroscience, 20, 6173- 6180.

Mandler, G. (1980). Recognizing: The judgment of previous occurrence. Psychological Review, 87, 252- 271.

Miller, E. K., \& Cohen, J. D. (2001). An integrative theory of prefrontal cortex function. Annual Review of Neuroscience, 24, 167- 202

Morris, C. D., Bransford, J. D., \& Franks, J. J. (1977). Levels of processing versus transfer appropriate processing. Journal of Verbal Learning and Verbal Behavior, 16, 519- 533.

Nairne, J. S. (1986). Active and passive processing during primary rehearsal. American Journal of Psychology, 99, 301- 314.

Naveh-Benjamin, M., \& Jonides, J. (1984). Maintenance rehearsal: A two-component analysis. Journal of Experimental Psychology: Learning, Memory, and Cognition, 10, 369- 385.

Otten, L. J., Henson, R. N., \& Rugg, M. D. (2001). Depth of processing effects on neural correlates of memory encoding: Relationship between findings from across- and within-task comparisons. Brain, 124, 399- 412.

Paller, K. A., Kutas, M., \& Mayes, A. R. (1987). Neural correlates of encoding in an incidental learning paradigm. Electroencephalography and Clinical Neurophysiology, 67, 360- 371.

Paulesu, E., Frith, C. D., \& Frackowiak, R. S. (1993). The neural correlates of the verbal component of working memory. Nature, 362, 342- 345.

Petrides, M. (1996). Specialized systems for the processing of mnemonic information within the primate frontal cortex. Philosophical Transactions of the Royal Society of London, Series B: Biological Sciences, 351, 1455- 1461.

Poldrack, R. A., Wagner, A. D., Prull, M. W., Desmond, J. E., Glover, G. H., \& Gabrieli, J. D. (1999). Functional specialization for semantic and phonological processing in the left inferior prefrontal cortex. Neuroimage, 10, 15- 35.

Price, C. J., Moore, C. J., Humphreys, G. W., \& Wise, R. S. J. (1997). Segregating semantic from phonological processes 
during reading. Journal of Cognitive Neuroscience, 9, 727- 733 .

Rowe, J. B., Toni, I., Josephs, O., Frackowiak, R. S., \& Passingham, R. E. (2000). The prefrontal cortex: Response selection or maintenance within working memory? Science, 288, 1656- 1660.

Rugg, M. D. (1995). Event-related potential studies of human memory. In M. S. Gazzaniga (Ed.), The cognitive neurosciences (pp. 789-801). Cambridge: MIT Press.

Rundus, D. (1971). Analysis of rehearsal processes in free recall. Journal of Experimental Psychology, 89, 63- 77.

Sandquist, T. F., Rohrbaugh, J. W., Syndulko, K., \& Lindsley, D. B. (1980). Electrophysiological signs of levels of processing: Perceptual analysis and recognition memory. Psychophysiology, 17, 568- 576.

Smith, E. E., \& Jonides, J. (1998). Neuroimaging analyses of human working memory. Proceedings of the National Academy of Sciences, U.S.A., 95, 12061- 12068.

Squire, L. R. (1992). Memory and the hippocampus: A synthesis from findings with rats, monkeys, and humans. Psychological Review, 99, 195- 231.

Talairach, J., \& Tournoux, P. (1988). Co-planar stereotaxic atlas of the buman brain. New York: Thieme.

Thompson-Schill, S. L., Aguirre, G. K., D'Esposito, M., \& Farah, M. J. (1999). A neural basis for category and modality specificity of semantic knowledge. Neuropsychologia, 37, 671- 676 .

Tulving, E. (1966). Subjective organization and effects of repetition in multi-trial free-recall learning. Journal of Verbal Learning and Verbal Behavior, 5, 193-197.

Tulving, E. (1983). Elements of episodic memory. New York: Oxford University Press.

Tulving, E. (1985). How many memory systems are there? American Psychologist, 40, 385- 398.

Wagner, A. D., Koutstaal, W., Maril, A., Schacter, D. L., \& Buckner, R. L. (2000). Task-specific repetition priming in left inferior prefrontal cortex. Cerebral Cortex, 10, 1176- 1184.

Wagner, A. D., Koutstaal, W., \& Schacter, D. L. (1999). When encoding yields remembering: insights from event-related neuroimaging. Philosophical Transactions of the Royal Society of London, Series B: Biological Sciences, 354, 1307- 1324.

Wagner, A. D., Maril, A., Bjork, R. A., \& Schacter, D. L. (in press). Multiple forms of executive control: fMRI evidence for functional dissociations within lateral prefrontal cortex. Neuroimage.

Wagner, A. D., Paré-Blagoev, E. J., Clark, J., \& Poldrack, R. A. (2001). Recovering meaning: Left prefrontal cortex guides controlled semantic retrieval. Neuron, 31, 329-338.

Wagner, A. D., Schacter, D. L., Rotte, M., Koutstaal, W., Maril, A., Dale, A. M., Rosen, B. R., \& Buckner, R. L. (1998). Building memories: Remembering and forgetting of verbal experiences as predicted by brain activity. Science, 281, 1188- 1191.

Woodward, A. E., Bjork, R. A., \& Jongeward, R. (1973). Recall and recognition as a function of primary rehearsal. Journal of Verbal Learning and Verbal Behavior, 12, 608- 617. 